\title{
ATP Depletion Impairs Activity-dependent Ultrafast Endocytosis at Hippocampal Synapses
}

Quan Gan ${ }^{1}$, Shigeki Watanabe ${ }^{1,2}$

1. Department of Cell Biology, Johns Hopkins School of Medicine, Baltimore, USA.

2. The Solomon H. Snyder Department of Neuroscience, Johns Hopkins School of Medicine, Baltimore, USA.

Glucose is the major energy source in neurons. Recent evidence suggests that inhibiting either glycolysis or oxidative phosphorylation results in a local depletion of ATP in presynaptic terminals, which impairs vesicle recycling during sustained synaptic transmission ${ }^{1,2}$. However, it is not clear which step in the synaptic vesicle recycling process is most critically limited by the availability of ATP, since ATP is required at multiple steps during this process including endocytosis, endosomal sorting, and refilling of neurotransmitters (Figure 1).

In this study, we used time-resolved electron microscopy to investigate the effect of ATP depletion on synaptic vesicle recycling on the ultrastructural level. As described previously ${ }^{3}$, cultured mouse hippocampal neurons expressing channelrhodopsin are stimulated by $10 \times 10 \mathrm{~ms}$ light pulses at $20 \mathrm{~Hz}$, and then rapidly frozen at high pressure after a delay period of $1 \mathrm{~s}$ to capture transient changes in membrane structures. Under these conditions, clathrin-independent ultrafast endocytosis is the predominant mechanism at the plasma membrane; synaptic vesicles are regenerated from endosomes by clathrin scaffolds (Figure 1) ) $^{3,4}$. In the control experiment, endocytic pits were fully internalized by $1 \mathrm{~s}$ post-stimulation, and thus the number of pits at this time point $(0.06 \pm 0.023$ per synaptic profile, mean $\pm \mathrm{SEM})$ is not significantly different from the number in the unstimulated control $(0.09 \pm 0.028 ; p=$ 0.42) (Figure 2C). In contrast, after $5 \mathrm{~min}$ of ATP depletion (10 $\mathrm{mM} 2$-deoxyglucose and $1 \mu \mathrm{M}$ oligomycin A), endocytic pits were trapped on the plasma membrane $1 \mathrm{~s}$ post-stimulation (Figure 2A). These pits were not coated by clathrin. In addition, they were found near or away from active zones (Figure 2A), indicating that these are likely ultrafast endocytic intermediates. The most notable feature of these pits was that they had a wide opening at the base, hinting at an inability to pinch off from the plasma membrane. Occasionally, a second pit was found "stacked" on another pit (Figure 2A, right panel), further supporting the idea that fission is perturbed. Pits with similar appearances were trapped at unstimulated synapses (Figure 2B), albeit at lower frequencies (no stimulation, $0.28 \pm 0.040$; after stimulation, $0.50 \pm 0.050, p=0.001)$. The number of pits trapped on the plasma membrane is lower when ATP is depleted for a shorter period $(3 \mathrm{~min}$ ) (Figure 2C), suggesting that synaptic vesicle endocytosis resulting from sporadic activity of neurons during the incubation period is also arrested by ATP depletion. Indeed, when spontaneous action potentials were blocked by $1 \mu \mathrm{M}$ TTX, the number of endocytic pits trapped at unstimulated synapses was much lower $(0.048 \pm 0.021)$. Our results suggest that ATP depletion arrests action potential-induced ultrafast endocytosis at a stage between pit formation and vesicle fission.

Based on our results, we speculate that the endocytic defects we observe is due to a dysfunction of dynamin caused by a shortage of GTP. In neurons, GTP cannot be produced from the TCA cycle and must be converted from ATP $^{5}$. A moderate perturbation in ATP level could lead to a severe depletion of GTP, which would in turn inhibit GTP-dependent processes such as the assembly of dynamin around the necks of endocytic pits (Figure 1) ${ }^{6}$. Further studies will be needed to test this hypothesis. 
References:

[1] Rangaraju, V., Calloway, N. and Ryan, T. A. Cell 156 (2014), p. 825.

[2] Pathak, D. et al, J. Biol. Chem 290 (2015), p. 22325.

[3] Watanabe, S. et al, Nature 504 (2013), p. 242.

[4] Watanabe, S. et al, Nature 515 (2014), p. 228.

[5] Dobolyi, A. et al, Brain Struct. Funct. 220 (2015), p. 135.

[6] Hinshaw, J. E. and Schmid, S. L. Nature 374 (1995), p. 190.

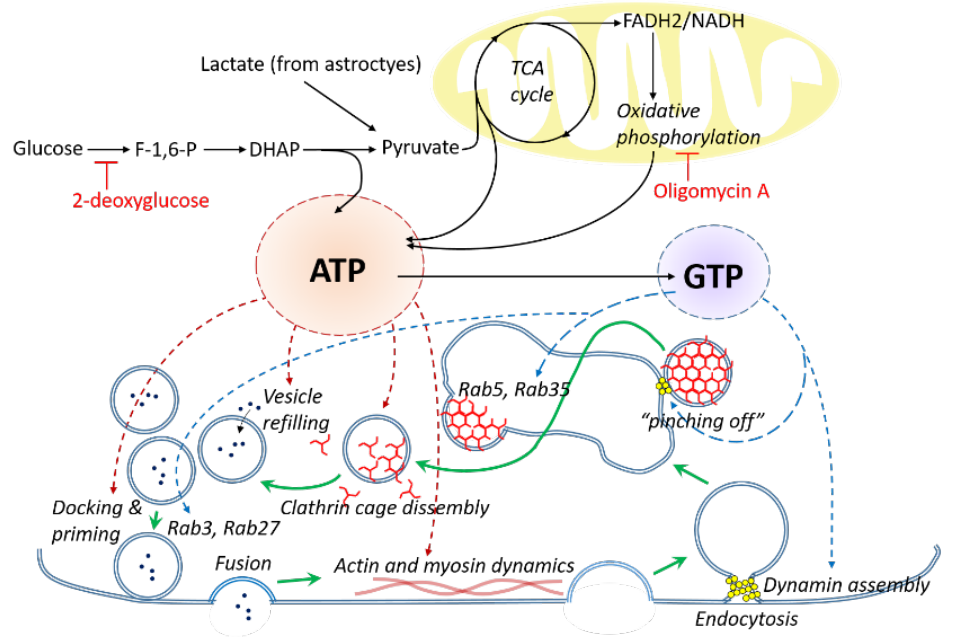

Figure 1. ATP is required at multiple stages of synaptic vesicle recycling. In neurons ATP is produced via the breakdown of glucose, while GTP is produced from ATP. Synaptic vesicle fusion is followed by ultrafast endocytosis, which requires actin for pit formation and dynamin for endocytic vesicle fission. Clathrindependent budding from endosomes regenerates new synaptic vesicles. Processes that require ATP and GTP are indicated by red and blue arrows, respectively.

Figure 2 ATP depletion arrests ultrafast endocytosis at an intermediate stage.

A) After 5 min of ATP depletion, neurons were stimulated 10 times at $20 \mathrm{~Hz}$. Non-coated endocytic pits were found trapped at the presynaptic plasma membrane $1 \mathrm{~s}$ poststimulation. Pits usually possessed a wide neck and were located either near or away from active zones (left and middle panels). "Stacked" pits were occasionally observed (right panel); B) Trapped pits were also found at unstimulated synapses following 5 min ATP depletion; C) The same experiments were done with shorter (3 min) or no ATP depletion. In one experiment, TTX was used to block spontaneous action potentials in unstimulated neurons. The number of trapped pits per synaptic profile was quantified for each experimental condition. Mann-Whitney U tests are performed and the resultant $p$ values are indicated.
2A
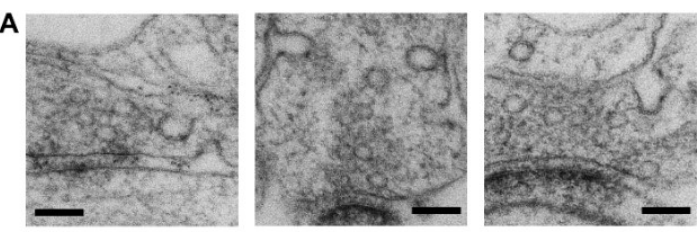

2B
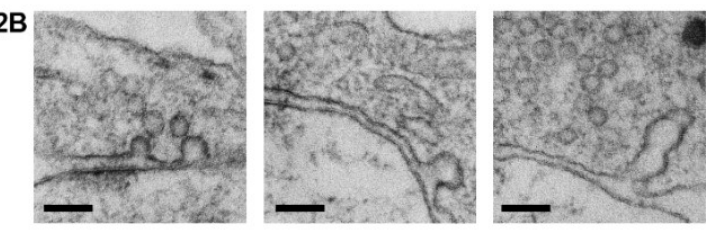

2C

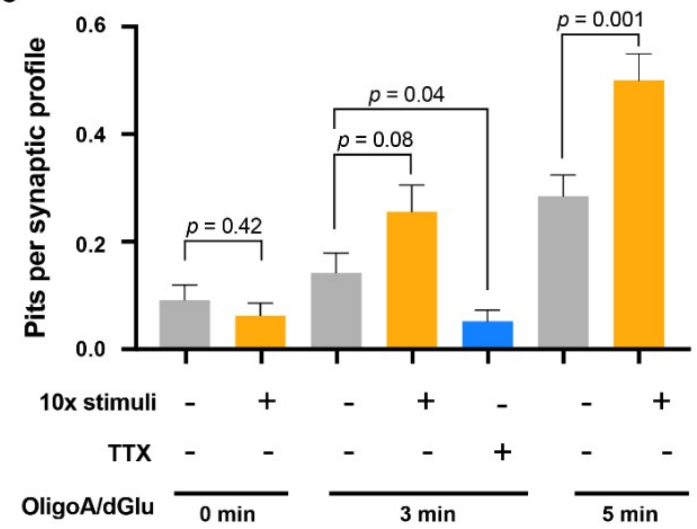

